\section{Stand Up act}

\section{By Tim Fulmer, Senior Writer}

In awarding a total of $\$ 73.6$ million to 5 teams of cancer researchers, not-for-profit Stand Up To Cancer was agnostic to specific therapeutic areas and instead focused on picking projects with the potential to enter the clinic within 3 years.

The funding model employed by the entertainment industrysponsored charity-providing three years of support with annual milestones-more closely resembles that of a venture firm than a government grant. Indeed, the average grant size of $\$ 14.7$ million is almost on par with the $\$ 16.8$ million average series A round for biotechs over the last five years, as tracked by BioCentury.

Stand Up To Cancer has enlisted the American Association for Cancer Research (AACR) to distribute the funds and evaluate each research team's progress. The selection of teams began last July when the AACR issued a request for ideas for translational cancer research projects. A 20 -member scientific advisory committee assembled by AACR reviewed proposals from 237 groups, eventually recommending 5 (see Table 1, "Stand Up To Cancer awards").

"The committee awarded the five research proposals that best hit the sweet spot of not being too preclinical on the one hand, and not having already made it into the clinic on the other," said committee member Raymond DuBois, who is provost and EVP of The University of Texas M.D. Anderson Cancer Center.

A key criterion guiding the selection of teams was sufficient expertise and experience in a given area to ensure that the new proposal stood a strong chance of entering the clinic by the third funding year if not sooner, he noted.

Another requirement of the grants was that each of the five research groups must consist of scientists drawn from multiple institutions and academic disciplines who have not previously worked together.

"By joining groups with complementary expertise who might not otherwise collaborate, the Stand Up To Cancer initiative hopes to accelerate translational cancer research," said AACR spokesman Jeremy Moore.

The groups will be required to meet with members of the advisory committee every 6-12 months to evaluate their progress and discuss potential roadblocks. Each team also will have to meet a number of prespecified research milestones, with teams held accountable if those milestones are not met. The financial terms of accountability would be worked out by the advisory committee on a case-by-case basis, Moore told SciBX.

Although the AACR declined to disclose the milestones, the leader of one of the teams, Daniel Haber, told SciBX that he and colleagues are expected "to optimize the technology over the first year, roll it out to our collaborating institutions in year two and initiate collaborative clinical trials in year three."

Haber is a professor of medicine at the Harvard Medical School and director of the Massachusetts General Hospital Cancer Center. His project is focused on developing a chip technology for detecting circulating tumor cells that could be used in cancer trials.

Table 1. Stand Up To Cancer awards. The charity will pay out $\$ 73.6$ million over 3 years to 5 groups of cancer researchers.

\begin{tabular}{l} 
Team leaders \\
Praig Thompson, Abramson Cancer Center of the University of \\
Daniel Von Hoff, The Translational Genomics Research Institute \\
Joe Gray, Lawrence Berkeley National Laboratory; \\
Dennis Slamon, University of California, Los Angeles \\
\hline Lewis Cantley, Beth Israel Deaconess Medical Center; \\
Charles Sawyers, Memorial Sloan-Kettering Cancer Center; \\
Gordon Mills, The University of Texas M.D. Anderson Cancer Center
\end{tabular}

Daniel Haber, Massachusetts General Hospital Cancer Center;

Mehmet Toner, Harvard Medical School

Research focus

Grant (\$M)

Use advanced imaging techniques to determine what nutrients pancreatic cancer cells require for growth and survival and run clinical trials designed to deprive pancreatic tumors of crucial nutrients in combination with standard chemotherapy

Develop therapies for the three major subtypes of breast cancer and design a discovery platform for identifying and validating new molecular targets and breast cancer drug combinations

Use biomarkers and imaging techniques to identify breast, ovarian and endometrial cancer patients who could benefit from phosphoinositide 3-kinase (PI3K) inhibitors and develop therapeutic combinations that hit multiple targets in cellular pathways that contribute to tumor growth

Optimize chip technology for detecting circulating tumor cells in a large-scale clinical setting and conduct clinical trials to assess the chip's ability to detect early cancers and monitor tumor treatment response

Stephen Baylin, The Sidney Kimmel Comprehensive Cancer Center; Identify biomarkers that can predict and monitor the efficacy of epigenetic cancer therapies, develop therapies that target cancer stem cells and design a clinical trial to test the efficacy of a secondgeneration epigenetic anticancer compound 


\section{TRANSLATIONAL NOTES}

Stand Up To Cancer raised the majority of its funds last September via a telethon that yielded $\$ 104$ million in donations. The 5 research teams will receive about $70 \%$ of that sum.

This year, the charity hopes to issue about $\$ 9$ million in a second round of grants that will provide up to $\$ 750,000$ over a 3 -year period to scientists who are early in their careers and whose research has translational potential.
Fulmer, T. SciBX 2(22); doi:10.1038/scibx.2009.896

Published online June 4, 2009

COMPANIES AND INSTUTIONS MENTIONED

American Association for Cancer Research, Philadelphia, Pa.

Harvard Medical School, Boston, Mass.

Massachusetts General Hospital Cancer Center, Boston, Mass.

Stand Up To Cancer, Los Angeles, Calif.

The University of Texas M.D. Anderson Cancer Center, Houston, Texas 